\title{
Stem Cell Dynamics and Cytoceuticals for Therapeutics and Toxicity Screening: A Review
}

\author{
Uche A Osunkwo ${ }^{1 *}$, Okolo $R U^{2}$, Bassi PU ${ }^{3}$, Ezeunala Mercy N ${ }^{4}$, Njoku Moses ${ }^{4}$ and Ben A Chindo ${ }^{1}$ \\ ${ }^{1}$ Department of Pharmacology \& Toxicology, National Institute for Pharmaceutical Research and Development, Nigeria \\ ${ }^{2}$ Department of Anatomical Sciences, College of Health Sciences, University of Abuja, Nigeria \\ ${ }^{3}$ Department of Pharmacology \& Therapeutics, College of Health Sciences, University of Abuja, Nigeria \\ ${ }^{4}$ Department of Microbiology and Biotechnology, National Institute for Pharmaceutical Research and Development, \\ Nigeria
}

\begin{abstract}
Summary
Cellular therapy involves use of whole cell or cell cytoplasmic macro-molecular inclusions and their derivatives as resources for treatment and management of diseases and their complications in animals and humans. The use of these biological and bio-medical resources in modern times is increasingly gaining popularity within the community of health care givers. This has become so in recent years mostly because of the ever increasing availability of new cell-derived therapeutic agents or cytoceuticals. These cytoceuticals of stem cellular origin are being developed from the painstaking research and manipulative developmental effort of bio-molecular scientists, curious and intuitive health care givers and the organized global business community including persons interested in regulatory ethics.
\end{abstract}

The diversity of multipotent stem cells and the availability of induced pluripotent stem cell (iPSC) reprogramming technologies have widened the possibility of fast-tracking personal regenerative medicine as well as reducing many fears inherent in ethical and religious concerns. Many tissues and organs of the animal or human body have been partially or wholly regenerated from stem cellular proliferation and differentiation. Therefore, cytoceuticals derivable from a combination of biological and non-biological materials (e.g. chemicals) will become abundant in the near future as they are being developed in various laboratories, and efficacy trials in clinical settings around the world are giving out their reports. Enhancement of circulatory stem cell populations using exogenous substances or by direct injection of cytoceuticals will give rise to novel pharmacological principles. Conceivably, cellular therapy has become a relevant part of curriculum development for training in health sciences and services. Ethical and regulatory principles of good laboratory practice as well as current good manufacturing practice will naturally apply to this emerging health-related discipline.

Toxicity screening being a relevant aspect of drug development prior to commercialization is currently taking a center stage. Therefore, acute, sub-acute and chronic toxicity screening modules have been developed using a variety of stem cell models. For example, using mouse spermatogonial stem cells, it has been demonstrated that hydroxyurea induces DNA damage and causes male reproductive toxicity [1,2]. Also, mouse embryonic stem cells can be used for testing for chemical mechanisms of disruption of differentiation and cell growth [3].

\section{Introduction}

The global quest for a slight paradigm shift from traditional clinical management of diseases pattern including alleviation of pain and suffering reminiscent of post "Materia medica" period to a technology-exploited evidence based personalized medicine in the current millennium cannot be over emphasized. Fontes and Thompson [4] and Osunkwo, [5] (review) and Yingai Shi and Yuanyuan Zhang [6] have pointed out that the high survival of stem cells in culture and their stability during proliferation and differentiation enables scientists to manipulate them for various types of cellular studies. However, all parties seem to agree that prior to exploitation of new technologies, there has to be strong evidence in favor of human and animal safety, efficacy and quality of cellular preparations, as well as marketability of products arising from adopted new technologies.

*Corresponding author: Uche A Osunkwo, Department of Pharmacology \& Toxicology National Institute for Pharmaceutical Research and Development, Abuja, Nigeria, Tel: +234-803-3296137

Accepted: January 25, 2020

Published online: January 27, 2020

Citation: Osunkwo UA, Okolo RU, Bassi PU, et al. (2020) Stem Cell Dynamics and Cytoceuticals for Therapeutics and Toxicity Screening: A Review. Insights Stem Cell Res Ther 2(1):15-22 
An improved understanding of the dynamics of pluripotent stem cells which started with embryonic stem cells (ESCs) reported to have been derived from human blastocyst [7] and progressed to induced pluripotent stem cells (iPSCs) has taken place mostly in the last twenty five years. Studying the dynamics of ESCS [8] revealed the capability of these cells to effect repair of cells and tissues of the animal body (see Review by $[9,10]$ ) as well as a capability to reversing the temporal changes associated with tissues and organs [11] see Review [12] to a large extent. Similarly, iPSCs $[8,13,14]$ have remained a recent biotechnology process from which human somatic cells [15] and body tissues have been produced including functional cardiomyocytes [16], hematopoietic and endothelial tissues [17], spinal muscular tissue [18], skin [13], liver and kidney [19] among many others.

According to Verma, et al. [10], genetic engineering, innovative cell culturing and induced pluripotency which generate various types of stem cells now make it possible to apply porcine stem cells for cardiac cell therapy and for innovative research. Moreover, the ready availability of stem cells for transplantation purposes encourages their application for clinical purposes. For example, Azzopardi and Blundell [20] have reported in their review article on umbilical cord stem cells that these cells can serve as a source of hematopoietic stem cells for transplantation. Jinhui Shen, et al. [21] also reported that peritoneal dialysis effluents can serve as a novel source of hematopoietic stem cells.

Attempts have been made by several scientists to define and characterize stem cells $[4,5,22]$. According to [22] stem cells are considered progenitor cells that can indefinitely (in appropriate medium) self-renew and differentiate giving rise to more specialized cells and tissue. These authors characterized stem cells by a multidisciplinary approach using morphology, expression of markers as observed by flow cytometry and fluorescence analysis, real time polymerase chain reaction, in addition to proliferation and differentiation [22]. For spermatogonial stem cells [5,23,24], they have been suggested to possess tremendous potential for treating many medical conditions $[6,25]$, have been reported to be isolated as pluripotent stem cells from neonatal mouse testis [26] and have also been reported to convert to pluripotent stem cells in-vitro [27].

Development of animal [23,28-34] or human [35-38] induced pluripotent stem cells (iPSC) is a major positive step that will enhance research and development on cellular bioactive materials (including organoid research technology). Cytoceuticals (as these new cellular materials may be called) derivable from various cells and tissues of human and animal bodies and applicable in clinical and experimental therapeutics are currently numerous and commercial interests involved in trials and evaluation have been reporting their findings in the last fifteen years. Organoids can be described as miniature organs elaborated from stem cells and progenitor cells which carry with them, and genetically express, the full complement of the genetic code of their main organs (see reviews by [39-41]. They are therefore made up of macromolecular materials derivable from within stem cells and they possess a potential to alter functionality of cells and tissues when co-cultured with exogenous substances like chemicals or biologicals like viruses. Already existing examples include organoids of brain, intestine, heart, etc. (see review by [42]. When the potential of these macromolecular compounds become beneficial to correction of disease states then they may be described as cytoceuticals (cellular therapeutic agents).

Acute and chronic safety concerns for these new cellular materials have variously been expressed. For example, in determining a developmental toxicity index, cellular differentiation process into neuronal precursor cells was reported to be disturbed following the addition of test chemicals into the culture space [43-47] see Review by [48] in his review of the use of stem cells for in vitro toxicity tests reported that stem cell derived hepatocyte and cardiomyocyte-like cells have featured prominently and that although a small catalog of chemical substances have been tested, validation studies that will enable application of this cytotoxicity results to human toxicity studies are yet to be done [48].

\section{Stem Cell Toxicity and Apoptosis}

Some cell types have been assessed for safety implications in both experimental and clinical settings. In a study [1] to assess hydroxy urea-induced toxicity, mouse spermatogonial stem cells were used. These investigators reported that hydroxy urea induced DNA damage, Reactive Oxygen Species (ROS) formation, and apoptosis in the spermatogonial stem cells thereby suggesting that mouse spermatogonial stem cells could be a useful model to predict male reproductive toxicity [1]. Another study [49] examined the possibility of manipulating the basement membrane of seminiferous tubules and the blood-testis barrier for the purposes of transducing viral particles into the microenvironment of spermatogonial stem cells. They found out that adeno-associated virus microinjected into the seminiferous tubules penetrated both the basement membrane and blood-testis barrier, transducing Sertoli cells, SSCs, peritubular cells and Leydig cells thereby allowing germline and niche manipulation [49].

It is important that specific mechanisms of cytotoxicity and apoptosis be looked into. Hexavalent chromium, $\mathrm{Cr}(\mathrm{VI})$, was reported to be cytotoxic to both male somatic cells and SSCs in a dose-dependent manner and also induced mitochondria-dependent apoptosis by inducing oxidative stress [50]. Futhermore, these authors stated that $\mathrm{Cr}(\mathrm{VI})$ is cytotoxic and impairs the physiological functions of male somatic cells and SSCs specifically by affecting the differentiation and self-renewal mechanisms of SSCs, disrupting steroidogenesis in TM3 cells, while in TM4 cells, the expression of tight junction signaling and cell receptor molecules was affected and the secretory functions were impaired [50]. Intratesticular injection of busulfan into mice prior to spermatogonial stem cell transplantation has been reported to be safe and non-toxic [51] suggesting that busulfan which causes hematopoietic toxicity, may be attenuating this untoward effect through a yet unknown mechanism.

In their study on the extent to which programmed ne- 
crosis (necroptosis) contributes to iPSCs induction, Al-Moujahed, et al. [52] reported that receptor-interacting protein kinase 3 was vital to iPSC reprogramming. This suggestion supports the notion that apoptosis-inducing caspases play a positive role in the process of iPSCs reprogramming.

On the other hand, the review of Shafique [2] points out the significance of using embryonic stem cells for the purposes of speeding up developmental toxicity testing and the possibility of a "Virtual embryo" for drug discovery and accelerated chemical risk assessment during testing for developmental toxicity. Earlier, Chandler and colleagues [3] had reported the use of embryonic stem cell adherent cell differentiation and cytotoxicity assay for the examination of many conventional toxic substances. These investigators reported that chemicals tested caused transcription based perturbation and impaired embryonic stem cell differentiation [3]. Finally, these authors [3] suggested that the results of their studies characterized metabolic and regulatory pathways as disruption mechanisms of some common environmental chemicals.

\section{Biocellular Materials and Mechanisms of Ac- tion of Cellular Products}

Stem cells are bio-cellular materials of animal origin from which other cells and tissues and organs are naturally derived. By natural in situ bio-engineering processes, stem cells can renew themselves (proliferation) and can also differentiate into all other types of cells (processes described as pluripotency, totipotency and multipotency). Through these natural bio-engineering processes, stem cells in the developing embryo, haematopoietic system, messenchymal tissue, neural network, cord blood, testicular tissue, retina and cancerous tissue have sustained the replacement of the temporal damages to the organs in which they are situated. The mechanistic action of stem cell materials in their attenuation of disease conditions has become of significant interest to many scientists. In a study of their intrinsic immunosuppressive properties, mesenchymal stem cells from iPSC were observed to alter phosphorylation and T cell-specific kinase, Protein Kinase $C$ theta, cellular localization thereby attenuating severity of the disease, and prolonging humanized mouse model survival [53].

Melatonin (an antioxidant) improves spermatogonial stem cells transplantation efficiency in azoospermic mice [54] and melatonin also relieves busulfan-induced spermatogonial stem cell apoptosis of mouse testis by inhibiting endoplasmic reticulum stress [55]. Furthermore, melatonin was investigated for its effects on the proliferation efficiency of neonatal mouse spermatogonial stem cells [56]. The authors reported that supplementation of their culture medium with $100 \mu \mathrm{M}$ melatonin significantly decreased reactive oxygen species (ROS) production in the treated group compared with the control group, and also increased SSC proliferation. From these reports, melatonin becomes an important molecule for use in manipulation of epigenetics, pluripotency, regenerative medicine and cell therapy.

The therapeutic potentials of biocellular materials of stem cell origin have been discovered in their ability to reproduce pancreatic islet cells for treatment of diabetes mellitus
[57]; haematopoietic cells for treatment of sickle cell disorders, blood-related diseases and HIV-AIDS; cardiomyocytes for treatment of cardiomyopathies and other heart diseases; neurons for treating Parkinsonison and Alzheimers disease, and hepatocytes that may become indicated in cirrhosis. Bioengineering technologies have also been advanced in many scientific laboratories in Europe and the United States of America and in China for the production of stem cell products for human and animal health, agricultural animal development, training and advancement of knowledge base.

Another example of the therapeutic potentials of biocellular materials of stem cell origin can be found in the laboratory development of retinal cells/tissue with high potential for use in regenerative treatment of eye diseases associated with damaged retina. [58] reported that patterns of gene and protein expression suggest involvement of human induced pluripotent stem cells in a process that may facilitate future therapies for retinal disorders. These authors called attention to the implication of their research result for cell replacement, pharmacological screening and disease modeling. Similarly, Ochiai, et al. [59] have demonstrated the capability to differentiate oral ectoderm from mouse embryonic stem cell. These authors also reported that biocellular materials (bone morphogenetic protein and fibroblast growth factor) enhanced oral ectoderm induction [59]. It is therefore, conceivable that focus on development of biocellular materials of stem cell origin will be worthwhile for future regenerative treatment of animal and human diseases.

Instances of use of stem cell content to influence stem cell functional activity for regeneration or wound healing and for organ well being abound. For surgical skin replacement, bone marrow-mesenchymal stem cells were reported to enhance healing of skin defect in rats [60]. In an attempt to characterize migration in a placental-derived chorionic mesenchymal stem cell line, [61] discovered that valproic acid increased migration of the mesenchymal stem cell line. These authors also reported that mesenchymal stem cell migration was associated with adherence to and transmigration through endothelial barrier [62]. Again, mesenchymal stem cells as well as exosomes released from mesenchymal stem cell have been reported to be supportive in treatment of osteoarthritis [63]. Similar observations have been made by Sagga, et al. [64] who reported that Limbal epithelial stem cells are involved in corneal epithelial maintenance and repair after injury. They made this suggestion following their surprising observation that injury to one eye causes a regenerative response in both eyes [64]. On the other hand, silver impregnated surgical dressing was reported to be associated with consistent culture and cell toxicity when compared with honey dressings on human skin keratinocytes and dermal fibroblasts [65].

Several studies and their reports have focused on the hematopoietic system in an attempt to elucidate the structural and functional capabilities of stem cells and their cytoplasmic constituents. Organoids derivable from somatic stem cells have also been studied. Hematopoietic stem and progenitor cells have been suggested to immunomodulate solid cancer development $[66,67]$. In a concise review on 
the modulation of cancer immunity with haematopoietic stem and progenitor cells, these authors write that these cells do regenerate entire blood and immune system in addition to being key modulators of cancer immunity, a property useful in cellular immunotherapy [67]. In like manner, the molecular mechanisms involved in inflammatory signals that control and sustain hematopoietic stem cell functions have been studied [68]. These authors reported that a deficiency in E3 ubiquitin ligase A20 caused loss of quiescence and compromised long-term hematopoietic regeneration, increased levels of Interferon- $\gamma$ signaling thereby suggesting a regulatory role for this enzyme in cell fate and quiescence of hematopoietic stem cells [68].

Animal agricultural development sector has witnessed largely profitable application of testicular stem cell research and development for products from bio-cellular materials. Manipulating the genetic resources (including deoxyribonucleic acid, DNA) of stem cells from selected animal species has enabled development of products for artificial insemination and programmed reproduction. Similarly, highly exotic and desirable characteristics of male farm animals (e.g. generous flesh and masculine agility in bulls) that are naturally stored in the genes of their testicular stem cells can now be preserved for a very long time and propagated as desired for agricultural development according to national or local policy guidelines.

Biochemical characterization of stem cells involving the use of enzymes (e.g. alkaline phosphatase or telomerase) and macromolecular aggregates e.g. receptors (Oct 3/4, Nanog, (D9) is important and has become widely acceptable. The use of these biochemical factors also promotes development and marketing of these biochemical compounds used also, as identification factors. For example, SOX4 which enhances neuronal differentiation has been reported to regulate transcription targets during neuronal development of neural stem cells [69] following oligodendrocyte differentiation. In effect, SOX4 enhancement of neuronal development in neural stem cells has been documented to be involved in reducing the expression of genes promoting oligodendrocyte differentiation. The use of morphological criteria (e.g. cellular shape and capsular luster, cellular aggregation or coloration and dynamics) and functional characteristics (e.g. in vitro expansion pattern, differentiation into new cells/tissues) and temporal relationships between developmental stages are equally important for advancement in stem cell research and application.

\section{Cytoceuticals and Disease Pathobiology}

Cytoceuticals can be experimentally derived or industrially manufactured from whole cells, subcellular particles, macrolecular aggregates of cell components or organic and inorganic effectors of stem cellular proliferation, aggregation and differentiation for the processes of tissue regeneration and replacement. A large number of simple and compound materials will be included in this list. However, it will be important to define and characterize each of them for the purposes of applying them for therapy of defined disease conditions.
Two disease conditions of significant pathobiological importance to stem cellular research are acquired immune deficiency syndrome (AIDS) secondary to Human Immunodeficiency virus (HIV) infection, and malaria (especially in endemic tropical regions). The HIV just like many other immunodeficiency viruses (e.g. Simian immunodeficiency virus) possesses well defined and largely characterized internal and external biochemical constituents that have been used as markers or target sites for drug action (and possible drug development effort). Sites like glycoprotein 120 is known to be associated with the virulence of HIV. These macromolecular sites may be considered useful for development of cytoceuticals.

\section{Stem Cell Therapy in HIV and AIDS}

The possibility strongly exists that stem cellular therapy can one day in the near future be available for clinical treatment of HIV infection and AIDS in man and variants in animals. This potential of cellular therapy in the treatment of this disease has been widened by observations in recent years on capability to obliterate infections by some types of stem cells on immunodeficiency virus infection complicating leukemia in patients. There has been a renewed interest in the possibility of using gene (a cytoplasmic and nuclear genetic inclusion of the cell) therapy in the treatment and eradication of HIV (Human immunodeficiency Virus) infection. This development is secondary to a report in the New England Journal of Medicine (February 2009) that a HIV positive patient with acute myeloid leukemia who had received a replacement of his stem cells with CD34+ peripheral stem cells from a matched unrelated donor (after allogeneic stem cell transplantation) was not detected with HIV after more than two years of being off antiretroviral and immunosuppressive drug treatment.

This scientifically exciting observation may be interpreted to be that at some stage of the development of the stem cell (that of the donor or those of the transplantation recipient), a potential macromolecular disposition emerges enabling the distinction of "self" macromolecular environment from the "non-self", identification of "non-self" macromolecular entity (e.g. HIV, particles, etc.) in the milieu and consequent elimination by destruction of the "non-self" macromolecular entity. There are other possibilities that can attempt to explain at cellular and molecular levels what happened to give rise to eradication of HIV from the "Berlin patient" for up to two years. Cellular, subcellular and biochemical mechanisms underlying the immunological bases of elimination of HIV from the circulation of a patient is certainly food for thought for those interested in cytoceuticals and cellular treatment of disease.

\section{Stem Cell Therapy in Malaria}

Let us examine closely, the issue of malaria in man and animals and the potential of cellular therapy in the treatment of this disease. In an unpublished report of our studies, we observed that rats infected with Plasmodium berghei berghei, when treated with spermatogonial stem cells exhibited gross malformation of the parasites in the rat blood. It is therefore imaginable that spermatogonial 
stem cells may be deforming mouse malaria parasites in vivo [70], unpublished observations). This possibility has been encouraged by the commentary of Wang, et al. [71] who quoted the report of the work done by Souza and colleagues. The authors found that transplantation of bone marrow-derived mesenchymal stromal cells increased survival, reduced parasitemia, decreased malaria pigment accumulation in the spleen, liver and kidney, elevated Kupffer cell count in liver, alleviated renal injury and lung inflammation, and improved lung mechanics in an experimental mouse model of cerebral malaria. Wang and his colleagues then suggested the potential of mesenchymal stromal cells therapy for the treatment of cerebral malaria [71].

In a similar fashion, parasitic organisms like Plasmodium species are known to possess some defining macromolecular aggregates (sites) that are continually being studied with a view to developing vaccines or other therapeutic agents targeting these sites. The pathogenicity of Plasmodium species has been reported to involve release of macromolecules that elicit induction of pyrexia. For example, cyclooxygenase inhibiting substances like acetyl salicylic acid or other prostaglandin modulators act as antipyretic chemical agents. It is noteworthy that there exists a temporal relationship in the elaboration of these macromolecules that form the biochemical basis of virulence, pathogenicity or therapeutic effectiveness. It is important to understand these temporal relationships so that stem cellular proliferative or differentiative activities can be related to them for cellular therapy in animals or humans.

\section{Cellular Therapy}

Cellular therapy may be a terminology preferred to cytotherapy mainly because single stem cell proliferative activity is known to culminate in aggregation of cells which can be directed to specific differentiation for the purposes of tissue regeneration and therapy of disease. Thus, single cells are not likely to effect therapy unless such cells have been clustered into biological tissues to regenerate diseased biological tissues. Cellular therapy and consequent regenerative medicine in animals or humans produce desired therapeutic effect often reminiscent of surgical resection and repair.

Pharmacological intervention in disease states focuses seriously on the underlying pathophysiological process which may be treated by such pharmacological intervention, for example, following biochemical or biophysical alteration of structure and function. Specifically, replacement of dopamine deficient neuronal cells with the same type of cells possessing dopamine efficiency will result in amelioration of some clinical symptoms in the Parkinson disease patient. In a similar way, cellular therapy will continue to rely on bio-pathological state e.g. damaged heart muscle or functionally defective $\beta$-cells of islets of Langerhans, the damaged cells of which can be replaced by newly elaborated and fully functional cluster of similar cells. Cellular therapy of a large number of experimental and clinical disease conditions has been effected and these include, diabetes mellitus (types 1 and 2), Parkinson's disease, etc.

\section{Cytoceuticals and Drug Discovery}

Cellular therapy will become complementary to drug therapy in this millennium for the treatment of life-threatening diseases. Cellular dynamics including systematic and organized in situ elaboration, release, reabsorption and sequestration of cytoplasmic and nuclear genetic inclusions and genetic materials form the bases of our understanding of basic cellular biology of development and embryogenesis that is exploited in drug discovery. Glicksman and her colleagues [72] have briefly reviewed stem cellular mechanisms in drug discovery. In their review of the therapeutic potentials of stem cells, Glicksman and her colleagues [72], have made reference to the possibility of "a small molecule drug" being capable of stimulating stem and progenitor cells in adult tissues such as skin, muscle, liver, lung, blood, fat, bone marrow, hair follicles and brain, to regenerate messenchymal stem cells and be harnessed to heal damaged tissues.

Reprogramming adult cells to create induced pluripotent stem cells (iPSCs), reverse differentiation of stem cells under unconducive environments, spontaneous reactivation for tumorigenesis as well as apoptosis secondary to unconducive environments are all aspects of the dynamics of cellular cytoplasmic and nuclear genetic inclusions. The creation of human and animal disease models and the testing of "small molecule drug" can be achieved using iPSCs in appropriately designed studies. These so-called small molecules could be part of the cytoplasmic milieu and may influence self renewal or differentiation of stem cells $[73,74]$.

It is of special interest to note that the reports of Takahashi and Yamanaka [8] and [13] on new methods of creating iPSCs have become more exciting for the possibility of exploiting cytoceuticals for drug discovery and development. Two major biotechnology industries (Stemgent and Fate Therapeutics) in their CATALYST alliance plan to maximize delivery of iPSC technology (free of viral reprogramming factors) for creation of models of human diseases, discovery and testing of drug candidates, and development of personalized cell therapies (www.worldstemcellsummit. com/chapters09/2-1_2009.pdf).

\section{New Pharmacological Principles}

Enhancement of circulatory stem cell populations using exogenous substances or by direct injection will give rise to novel pharmacological principles. It has been argued that general health correlates well with the levels of circulating stem cell populations in blood. One method of enhancing circulatory stem cells and effecting visible structural and functional changes to human and animal somatic systems would be reversal of senescence (see Reviews $[12,75]$. The results of a study on the effects of senescence and high fat diet (a risk factor for many diseases) on lung epithelial and stem cells when compared to low fat diet in younger animals showed that both aging and high fat diet groups were associated with a significant increase in lung inflammation [76]. 
In a review on the clinical importance of umbilical cord stem cells, Alli and Al-Mulla [77] pointed out the possibility of umbilical cord blood stem cells as treatment materials for many diseases and disorders. Recently, enzyme activity involved macromolecular lead towards new drug discovery are beginning to emerge. In a classic study, Koutsouraki, et al. [78] have suggested a role for 2-oxoglutarate-dependent dioxygenases in hypoxia's promotion of undifferentiated human embryonic stem cells (hESC) induction and self-renewal. These investigators further suggested that a better comprehension of the relevance of reduced oxygen environment $(0.5$ to $5 \% \mathrm{O}_{2}$ ) to human embryonic stem cells proliferation will augur well for cell culture and (hESC) studies.

\section{Statement on Conflict of Interest by the Re- search Team}

Nothing declared.

\section{Acknowledgment}

We wish to acknowledge the support of Nigerian government tertiary education trust fund (TETFUND) Institution (University of Abuja, Abuja) based research grant No. 6 of 2015 awarded to Principal Investigator, Prof. Uche A. Osunkwo.

\section{References}

1. Hye Lyun Jeon, Jung-Sun Yi, Tae Sung Kim, et al. (2017) Development of a test method for the evaluation of DNA damage in mouse spermatogonial stem cells. Toxicol Res 33: 107-118.

2. Shafique Sidra (2018) Preclinical developmental toxicity testing and advanced in-vitro stem cell based systems. Timely Top Clinical Immunol 2: 3-5.

3. Chandler KJ, Marianne Barrier, Susan Jeffay, et al. (2011) Evaluation of 309 environmental chemicals using a mouse embryonic stem cell adherent cell differentiation and cytotoxicity assay. PLOS ONE 6: 18540.

4. Fontes A Paula, Thompson W Angus (1999) Stem cell technology. Br Med J 319: 1308.

5. Osunkwo UA, N Ezeunala Mercy, M O Duru Osunkwo, et al. (2009) A comparative study of the gross structure and distribution of spermatogonia-like cells in the testis of pre-pubertal and mature adult rats. Journal of Cell and Animal Biology 3: 202-207.

6. Shi Yingai, Zhang Yuanyuan (2011) Mesenchymal stem cells for cell therapy and tissue regeneration in urology. In: Daniel Ebarli, Regenerative medicine and tissue engineering-Cells and Biomaterials. IntechOpen, London, UK.

7. Thompson BJ, Plunkett SP, Gurman JB, et al. (1998) SOHO/EIT observations of an Earth-directed coronal mass ejection on May 12, 1997. Geophysical Research Letters 5: 2461-2464.

8. Takahashi K, Yamanaka S (2006) Induction of pluripotent stem cells from mouse embryonic and adult fibroblast cultures by defined factors. Cell 126: 663-676.

9. Julio C Davila, Gabriela G Cezar, Mark Thiede, et al. (2004) Use and Application of Stem Cells in Toxicology. Toxicological Sciences 79: $214-223$

10. Verma V, Mehta A, Pal S, et al. (2014) In pursuit of porcine pluripotent stem cells for autologous cell therapy. Stem Cell Discov- ery 4: 107-124.

11. Silver H, Conboy IM (2008) Aging and stem cell renewal. StemBook.

12. Osunkwo UA (2013) Cellular drugs of the future and reversal of aging A micro Review. Advances in Science and Technology 7: 12-17.

13. Takahashi K, Tanabe K, Ohnuki M, et al. (2007) Induction of pluripotent stem cells from adult human fibroblasts by defined factors. Cell 131: 861-872.

14. Okita K, Ichisaka T, Yamanaka S (2007) Generation of germline-competentinduced pluripotent stem cells. Nature 448: 313317.

15. Yu J, Vodyanik MA, Smuga-Otto K, et al. (2007) Induced pluripotent stem cell lines derived from human somatic cells. Science 318: 1917-1920.

16. Zhang J, Wilson GF, Soerens AG, et al. (2009) Functional cardiomyocytes derived from human induced pluripotent stem cells. Circ Res 104: 30-41.

17. Choi KD, Yu J, Smuga-Otto K, et al. (2009) Hematopoietic and endothelial differentiation of human induced pluripotent stem cells. Stem Cells 2: 559-567.

18. Ebert AD, Yu J, Rose FF, et al. (2009) Induced pluripotent stem cells from a spinal muscular atrophy patient. Nature 457: 277280.

19. Abu-Zinadah OA, Hussein HK (2011) The potential of stem cells to correct the lost tissue mass in liver and kidney of rat after copper toxicity. Insight Stem Cell Research 1: 1-5.

20. Azzopardi JI, Blundell R (2018) Review :Umbilical cord stem cells. Stem Cell Discovery 8: 1-11.

21. Jinhui Shen, Junke Zheng, Ramesh Saxena, et al. (2015) Novel source of human hematopoietic stem cells from peritoneal dialysis effluents. Stem Cell Research 15: 299-304.

22. Gargiulo C, Pham VH, Hai NT, et al. (2015) Isolation and characterization of multipotent and pluripotent stem cells from human peripheral blood. Stem Cell Discovery 5: 19-32.

23. De Rooij DG, Van Pelt AMM (2003) Spermatogonial stem cell biology. ARBS Annu Rev Biomed Sci 5: 105-114.

24. Osunkwo UA, Ezeunala, Mercy N, et al. (2011) Optical microscope gross characterization of rat neonatal testicular germline stem cell expansion in vitro. International Journal of Genetics and Molecular Biology 3: 39-44.

25. Nayernia K (2007) Stem cells derived from testis show promise for treating a wide variety of medical conditions. Cell Res 17: 895-897.

26. Kanatsu-Shinohara M, Inoue K, Lee J, et al. (2004) Generation of pluripotent stem cells from neonatal mouse testis. Cell 119: 1001-1012.

27. Conrad S, Renninger M, Hennenlotter J, et al. (2008) Generation of pluripotent stem cells from adult human testis. Nature 456 : 344-349.

28. Ogawa T, Arechaga JM, Avarbock MR, et al. (1997) Transplantation of testis germinal cells into mouse seminiferous tubules. Int J Dev Biol 41: 111-122.

29. Brinster RL, Nagano M (1998) Spermatogonial stem cell transplantation, cryopreservation and culture. Semin Cell Dev Biol 9: 401-409. 
Citation: Osunkwo UA, Okolo RU, Bassi PU, et al. (2020) Stem Cell Dynamics and Cytoceuticals for Therapeutics and Toxicity Screening: A Review. Insights Stem Cell Res Ther 2(1):15-22

30. Ryu BY, Orwig KE, Avarbock R Mary, et al. (2003) Stem cell and niche development in the post natal rat testis. Dev Biol 263: 253263.

31. Ryu Buom-Yong, Orwig KE, Oately JM, et al. (2006) Effects of aging and niche microenvironment on spermatogonial stem cell self-renewal. Stem Cells 24: 1505-1511.

32. Kubota H, Brinster RL (2006) Technology insight in vitro culture of spermatogonial stem cells and their potential therapeutic uses. Nat Clin Practice Endocrinol Metabolism 2: 99-108.

33. Zhang Z, Shao S, Meistrich ML (2006) Irradiated mouse testes efficiently support spermatogenesis derived from donor germ cells of mice and rats. J Androl 27: 365-375.

34. Zhang Z, Shao S, Meistrich ML (2007) The irradiation-induced block in spermatogonial differentiation is due to damage to the somatic environment not the germ cells. J Cell Physiol 211: 149158.

35. Jamala Mohamed, Sara L Lewandowskia, Matthew L Lawtona, et al. (2018) Derivation and characterization of putative craniofacial mesenchymal progenitor cells from human induced pluripotent stem cells. Stem Cell Research 33: 100-109.

36. Haasea Alexandra, Wolfgang Glienke, Lena Engels, et al. (2019) GMP-compatible manufacturing of three iPS cell lines from human peripheral blood. Stem Cell Research 35: 101394.

37. Zuzana Kahounová, Eva Slabákováa, Lucia Binóa, et al. (2019) Generation of human iPSCs from fetal prostate fibroblasts HPrF . Stem Cell Research 35: 101405.

38. Khabarova AA, Pristyazhnyuk IE, Nikitina TV, et al. (2019) Induced pluripotent stem cell line ICAGi001-A derived from human skin fibroblasts of a patient with 2 p25.3 deletion and 2p25.3-p23.3 inverted duplication. Stem Cell Research 34: 101377.

39. Beatrice Xuan Ho, Nicole Min Qian Pek, Boon-Seng Soh (2018) Disease modeling using 3d organoids derived from human induced pluripotent stem cells. Int J Mol Sci 19: 936.

40. Xuyu Qian, Hongjun Song, Guo-li Ming (2019) Brain organoids advances applications and challenges. Development 146: 1-12.

41. Lehmanna Ruth, Connie M Lee, Erika C Shugartc, et al. (2019) Human organoids a new dimension in cell biology. Molecular Biology of the Cell 30: 1129-1137.

42. McCauley Heather A, James M Wells (2017) Pluripotent stem cell-derived organoids using principles of developmental biology to grow human tissues in a dish. Development 144: 958-962.

43. Shinde V, Klima S, Sureshkumar PS, et al. (2015) Human pluripotent stem cell based developmental toxicity assays for chemical safety screening and systems biology data generation. J Vis Exp 100: 52333

44. Rempel E, Hoelting L, Waldmann T, et al. (2015) A transcriptome-based classifier to identify developmental toxicants by stem cell testing: design validation and optimization for histone deacetylase nhibitors. Arch Toxicol 89: 1599-1618.

45. Pallocca G, Grinberg M, Henry M, et al. (2016) Identification of transcriptome signatures and biomarkers specific for potential developmental toxicants inhibiting human neural crest cell migration. Arch Toxicol 90: 159-180.

46. Shinde V, Hoelting L, Srinivasan SP, et al. (2017) Definition of transcriptome-based indices for quantitative characterization of chemically disturbed stem cell development introduction of the STOP-Toxukn and STOP-Toxukk tests. Arch Toxicol 91: 839-864.
47. Colaianna M, Ilmjärv S, Petersonm H, et al. (2017) Fingerprinting of neurotoxic compounds using a mouse embryonic stem cell dual luminescence reporter assay. Arch Toxicol 91: 365-391.

48. Herman M Bolt (2017) Stem cells in toxicological research. Archives of Toxicology 91: 4029-4030.

49. Satoshi Watanabe, Mito Kanatsu-Shinohara, Narumi Ogonuki, et al. (2018) In vivo genetic manipulation of spermatogonial stem cells and their microenvironment by adeno-associated viruses. Stem Cell Reports 10: 1551-1564.

50. Joydeep Das, Min-Hee Kang, Eunsu Kim, et al. (2015) Hexavalent chromium induces apoptosis in male somatic and spermatogonial stem cells via redox imbalance. Sci Rep 5: 13921.

51. Qin $Y$, Liu L, He Y, et al. (2016) Testicular busulfan injection in mice to prepare recipients for spermatogonial stem cell transplantation is safe and non-toxic. PLoS One 11: 0148388.

52. Al Moujahed, Ahmad B Tiana, Nikolaos E Efstathiou, et al. (2019) Receptor interacting protein kinase 3 (RIP3) regulates iPSCs generation through modulating cell cycle progression genes. Stem Cell Research 35: 101387-101394.

53. Ozaya E Ilker, Jyothi Vijayaraghavan, Gabriela Gonzalez-Perez, et al. (2019) Cymerus $^{\mathrm{TM}}$ iPSC-MSCs significantly prolong survival in a pre-clinical, humanized mouse model of Graft-vs-host disease. Stem Cell Research 35: 101401-101414.

54. Gholami M, Saki G, Hemadi M, et al. (2014) Melatonin improves spermatogonial stem cells transplantation efficiency in azoospermic mice. Iran J Basic Med Sci 17: 93-99.

55. Yanhua Cuia, Lipeng Bo, Jia Fanga, et al. (2017) Melatonin relieves busulfan-induced spermatogonial stem cell apoptosis of mouse testis by inhibiting endoplasmic reticulum stress. Cell Physiol Biochem 44: 2407-2421.

56. Shadan Navid, Mehdi Abbasi, Yumi Hoshino (2017) The effects of melatonin on colonization of neonate spermatogonial mouse stem cells in a three-dimensional soft agar culture system. Stem Cell Research \& Therapy 8: 233.

57. Majeed C, Al Shammari AM, Yausif EH, et al. (2015) BM-MSCs differentiated insulin-producing cells produce more insulin in presence of EGF than of FGF. Stem Cell Discovery 5: 33-39.

58. Sridhar A, Steward M, Melissa Meyeret, et al. (2013) Nonxenogeneic Growth and Retinal Differentiation of human induced pluripotent stem cells. Stem Cells Translational Medicine 2: 255-264.

59. Ochiai Hiroshi, Hidetaka Suga, Tomiko Yamada, et al. (2015) BMP4 and FGF strongly induce differentiation of mouse ES cells into oral ectoderm. Stem Cell Research 15: 290-298.

60. Ghada F Mohamed, Manal H Moussa, Sahar Omar, et al. (2019) Therapeutic role of mesenchymal stem cells seeded dermal matrix versus acellular dermal matrix in healing of skin defect. J Stem Cell Research \& Therapeutics 5: 13-21.

61. Qin SQ, Kusuma GD, Al-Sowayan B, et al. (2016) Establishment and characterization of fetal and maternal mesenchymal stem/ stromal cell lines from the human term placenta. Placenta 39: 134-146.

62. Al Sowayan B, Keogh RJ, Abumaree M, et al. (2019) Valproic acid stimulates in vitro migration of the placenta-derived mesenchymal stem/stromal cell line CMSC29. Stem Cell Investig 6: 3-11.

63. Sheykhhasan M, Manoochehri H, Pourjafar M, et al. (2018) Mesenchymal stem cells as a valuable agent in osteoarthritis treatment. Stem Cell Investig 5: 41. 
64. Saggaa Nada, Lucia Kuffováa, Neil Vargesson, et al. (2018) Limbal epithelial stem cell activity and corneal epithelial cell cycle parameters in adult and aging mice. Stem Cell Research 33: 185198.

65. DuToit DF, BJ Page (2009) An invitro evaluation of the cell toxicity of honey and silver dressings. J Wound Care 18: 383-389.

66. King KY, Goodell MA (2011) Inflammatory modulation of HSCs Viewing the HSC as a foundation for the immune response. Nat Rev Immunol 11: 685-692.

67. Wildes Tyler J, Catherine T Flores, Duane A Mitchell (2019) Concise review modulating cancer immunity with hematopoietic stem and progenitor cells. Stem Cells 37: 166-175.

68. Nakagawa Masahiro Marshall, Harry Davis, Chozha Vendan Rathinam (2018) A20 deficiency in multipotent progenitors perturbs quiescence of hematopoietic stem cells. Stem Cell Research 33: 199-205.

69. Braccioli L, Stephin J Vervoortb, Gianmarco Pumab, et al. (2018) SOX4 inhibits oligodendrocyte differentiation of embryonic neural stem cells in vitro by inducing Hes 5 expression. Stem Cell Research 33: 110-119.

70. Osunkwo UA (2012) Unpublished laboratory observation.

71. Wei Wang, Hui Qian, Jun Cao (2015) Stem cell therapy a nov- el treatment option for cerebral malaria? Stem Cell Research \& Therapy 6: 141 .

72. Glicksman Marcie, Lam Kelvin, Sittampalam G, et al. (2009) The use and promise of stem cells in drug discovery. World Stem Cell Report. Genetics Policy Institute. 45-48.

73. Emne N, Coleman R, Ding S (2007) A chemical approach to stem cell biology. Curr Opin In Chem Biol 11: 252-258.

74. Desbordes SC, Plakantonakis DC, Ciro D, et al. (2008) High thoroughput screening assay for the identification of compounds regulating self-renewal and differentiation in human embryonic stem cells. Cell Stem Cell 2: 602-612.

75. Pollina EA, Brunet A (2011) Epigenetic regulation of aging stem cells. Oncogene 30: 3105-3126.

76. Hegab AE, Mari Ozakia, Fatma Y Meligya, et al. (2018) High fat diet activates adult mouse lung stem cells and accelerates several aging-induced effects. Stem Cell Research 33: 25-35.

77. Ali Hamad, Fahd Al Mulla (2012) Defining umbilical cord blood stem cells. Stem Cell Discovery 2: 9.

78. Koutsourakia Eirini, Steve Pells, Paul A De Sousa (2019) Sufficiency of hypoxia-inducible 2-oxoglutarate dioxygenases to block chemical oxidative stress-induced differentiation of human embryonic stem cells. Stem Cell Research 34: 101358. 